\title{
Is hybrid SPECT/CT necessary for pre- interventional 3D quantification of relative lobar lung function?
}

\author{
Daniela Knollmann ${ }^{1}$, Jerome Avondo ${ }^{2}$ and Wolfgang M. Schaefer ${ }^{1 *}$ (D)
}

\author{
* Correspondence: wolfgang. \\ schaefer@mariahilf.de \\ ${ }^{1}$ Kliniken Maria Hilf, Nuclear \\ Medicine, Viersener Str. 450, 41063 \\ Moenchengladbach, Germany \\ Full list of author information is \\ available at the end of the article
}

\begin{abstract}
Background: In pulmonary malignancies pre-interventional 3D estimation of relative lobar perfusion is established to predict post-interventional functional outcome particularly in patients with borderline lung function. Aim was to test whether quantification from SPECT-scanners (non-hybrid) is as accurate as from SPECT/CTscanners (hybrid) when using dedicated software.

Methods: Sixty-one patients suffering from pulmonary tumours underwent lung SPECT/CT using Tc-99m MAA to predict postoperative residual lung function prior to surgical treatment. Quantification was done using "HERMES Hybrid 3D-Lung Lobe Quantification". In the hybrid approach SPECT and combined lowdoseCT/ diagnosticCT were used. In the non-hybrid approach SPECT and diagnosticCTs were used, lowdoseCTs were omitted. Bland Altman analysis was done to test for agreement.
\end{abstract}

Results: Three hundred five lobes were quantified. Evaluation time was 6:37 \pm 0 . 55 min (hybrid) versus 6:34 \pm 0.51 min (non-hybrid). Mean lobar value was $20.0 \pm 10$. $5 \%$ (range from 0 to 55\%) for hybrid and $20.0 \pm 10.6 \%$ (range from 0 to 58\%) for the non-hybrid approach, mean absolute difference was $1.31 \%$, no significant differences were found when analysing all values $(p>0.9)$. Correlation was excellent $(R=0.984$, slope of the regression line $1.001(p<0.0001))$. Intraclass correlation coefficient was 0 . 9843. Bland Altman limits were $-3.67 \%$ and $3.67 \%$.

Conclusion: Excellent concordance was found for 3D-quantification of relative lung perfusion when comparing a hybrid vs. non-hybrid approach. Using sophisticated software combining the generally available diagnosticCT and conventional SPECTdata reliable results for lobar perfusion can be obtained without the need for costly investment of SPECT/CT systems for this clinical question.

Keywords: Quantitative lung perfusion SPECT/CT, 3D quantification, Estimated postoperative FEV 1.0

\section{Background}

In pulmonary malignancies preoperative quantification of relative pulmonary lobar perfusion fraction using scintigraphy is an established procedure in predicting lung function after surgery (Bria et al., 1983; Markos et al., 1989). Functional preoperative evaluation of lung resection candidates is essential to assess the individual pulmonary and surgical risk (Bolliger \& Perruchoud, 1998) particularly in patients with borderline

(c) The Author(s). 2018 Open Access This article is distributed under the terms of the Creative Commons Attribution 4.0 International License (http://creativecommons.org/licenses/by/4.0/), which permits unrestricted use, distribution, and reproduction in any medium, provided you give appropriate credit to the original author(s) and the source, provide a link to the Creative Commons license, and indicate if changes were made. 
lung function (Brunelli et al., 2009). Traditionally, quantification was performed using planar images and a mathematical model (Wu et al., 2002); (Win et al., 2006; Giordano et al., 1997; Mende et al., 1990)) lacking accuracy in regard to individual differences and variants of pulmonary anatomy and lobe delineation especially in anatomy altering gross lung tumours. After the introduction of SPECT in the 1990s first attempts were made to use a 3D approach to overcome the above mentioned limitations but were hampered by the use of complex procedures, available only at single sites (Imaeda et al., 1995; Ohno et al., 2011; Suga et al., 2004).

Recent data have shown the superiority of the 3D approach using CT data for lobe delineation in comparison to planar imaging in calculation of relative lobar perfusion (Knollmann et al., 2015; Toney et al., 2014). In the last years hybrid SPECT/CT machines have become more available offering the opportunity to overcome the problem of lung lobe delineation by implementing CT data in the quantification workflow. Data from hybrid SPECT/CT-scanners allow for coregistration of the acquired low-dose CT to a diagnostic CT. This is available in virtually all patients with pulmonary malignancies and can be used as the best basis for anatomical lobe delineation.

The availability of hybrid SPECT/CT cameras is yet still limited often due to higher costs regarding purchase and maintenance resulting in the continued use of planar quantification. Nonetheless, in a relevant number of patients, lung perfusion SPECT data and a diagnostic CT are available. This provides the opportunity to perform 3D instead of planar derived quantification if a dedicated software algorithm for lung quantification is commercially available and allows for coregistration of separately acquired diagnostic CT and perfusion SPECT data.

The aim of the study was therefore to test whether CT based lung perfusion quantification from conventional SPECT-scanners (non-hybrid) is as exact as from SPECT/ CT-scanners (hybrid) when using a dedicated software algorithm.

\section{Methods}

Patients

In 2016 a total number of 96 patients was referred to our department for routine V/ P-SPECT/CT to predict postoperative residual lung function prior to surgical treatment or endoscopic procedures. 74 patients suffered from pulmonary tumours and 22 from emphysema or COPD. Since the study focuses on lung tumours the data of the 22 patients with emphysema or COPD as indication for the procedure were not included into the analysis.

Thirteen patients were excluded from this evaluation study due to the following reasons: previous pulmonary surgery (7 patients), lack of a timely diagnostic CT (2 patients), present tracheal cannula (2 patients), diagnostic CT in prone positioning (1 patient) and impossibility of segmentation of the diagnostic CT by the software algorithm (1 patient).

The remaining group of 61 patients comprised of 39 men and 22 women with a mean age of $70.4 \pm 8.1$ years (range $52-87$ years).

\section{Diagnostic CT}

Sixty-one full-dose CTs were available from different institutions (53 of those CTs were with contrast media). Since some patients brought their CT with them not in all cases 
tube voltage, CTDIvol or DLP were available. Therefore we report the mean values from the available data. Mean tube voltage (for 61/61 pts) was $119.0 \pm 5.4 \mathrm{kV}$, mean CTDIvol (for 53/61 pts) was $8.22 \pm 4.60$, mean DLP (for 53/61 pts) was $341.1 \pm$ $232.9 \mathrm{mGy}^{*} \mathrm{~cm}$, resulting mean dose (53/61 pts) was $5.58 \pm 3.29 \mathrm{mSv}$.

\section{Lung perfusion SPECT/CT}

Perfusion SPECT/CT was performed using a double-head gamma camera (Siemens SymbiaT, Siemens Medical Systems, Hofmann Estates, IL, USA) equipped with low-energy high-resolution collimators. Before perfusion scanning, a ventilation scan was acquired in each patient to exclude relevant pulmonary embolism. A minimum fourfold higher count rate was guaranteed for the perfusion compared to the ventilation scan.

SPECT acquisition started after intravenous injection of about $160 \mathrm{MBq}$ of Tc-99m labelled macroaggregated albumin (MAA) in upright position. Upright position was chosen since all pulmonary function tests - like spirometry - are done in upright position and we intend to predict the postoperative outcome for the patients in daily life in upright position. A $360^{\circ}$-acquisition in supine position with 32 projections, $8 \mathrm{~s}$ per projection and a matrix of $128 \times 128$ was used. Finally, a low-dose CT was performed (5 mm slice thickness; $110 \mathrm{kV}$ ). For the low-dose CT mean current was $22.7 \pm 5.9 \mathrm{mAs}$, mean CTDIvol was $1.32 \pm 0.34$, mean DLP was $40.5 \pm 11.3 \mathrm{mGy} * \mathrm{~cm}$, resulting mean dose was $0.66 \pm 0.16 \mathrm{mSv}$.

During SPECT/CT acquisition all patients were allowed to breathe shallowly; no respiratory gating was performed.

Images were reconstructed without any information from the low-dose CT using 3D iterative reconstruction with scatter correction (Flash 3D, 4 iterations and 8 subsets) and were then transferred to a Siemens syngo workstation with MI application software (Siemens Medical).

\section{D quantification of lobar perfusion}

For this retrospective study two different approaches concerning the CT based quantification of lung perfusion were pursued, evaluation time needed was counted for all quantifications.

In the hybrid approach SPECT (see Fig. 1) perfusion data were quantified after low-dose CT from the SPECT/CT camera was used for coregistration of the externally performed diagnostic CT.

In the non-hybrid approach (see Fig. 1) only perfusion SPECT data from the SPECT/ CT camera and an externally performed diagnostic full-dose CT were used for quantification, the low-dose CT was omitted in this approach as if no hybrid equipment was available.

As a first step in the quantification process perfusion SPECT, co-acquired low-dose CT data and diagnostic CT were transferred to commercially available software (HERMES Hybrid 3D - Lung Lobe Quantification, HERMES Medical Solutions, Stockholm, Sweden).

The software allowed the hybrid and non-hybrid approaches to be processed using the same step by step wizard workflow. The coregistration was performed automatically 


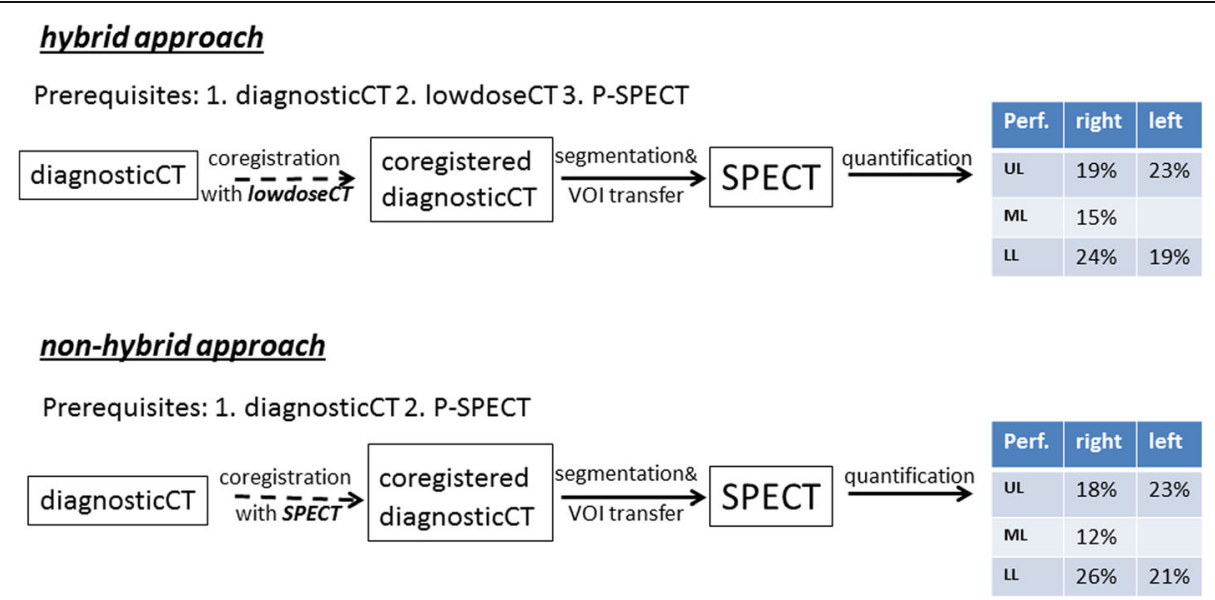

Fig. 1 Flow charts of the hybrid and the non-hybrid approach for lung perfusion SPECT segmentation and quantification (results are from patient from Fig. 2)

by the software. The hybrid approach coregistered the diagnostic CT with the low-dose CT whilst the non-hybrid approach coregistered the diagnostic CT with the SPECT perfusion. The software used a rigid (translation only) mattes mutual information algorithm for both approaches. The coregistration algorithm parameters were identical for both approaches. The manual uniform scaling adjustment was used to compensate for breathing mis-match of the diagnostic CT. For the hybrid method 37 cases out of 61 had no or only little manual adjustments, 24 cases out of 61 had relevant manual adjustments. For the non-hybrid method 29 cases out of 61 had no or only little manual adjustments, 32 cases out of 61 had relevant manual adjustments.

The right and left lung volumes were computed from the diagnostic CT using an automatic segmentation algorithm. The lung volumes were split into lobar regions by marking 5 points in 3 slices for each fissure. The resulting lobar regions were automatically transferred to the SPECT perfusion and the relative distribution of perfusion per lobar region was computed (workflow see Fig. 2).

\section{Data analysis}

Statistical analyses were done using Origin 6.1 G (OriginLab Corp., Northampton, England) and MedCalc (MedCalc Software, Ostend Belgium). Mean values \pm standard deviation of relative lobar perfusion were calculated from the hybrid approach and from the non-hybrid approach. Additionally mean processing times were calculated for both approaches. Data were tested for significant differences using the t-test for paired samples. A $p$-value $<0.05$ after Bonferroni correction for multiple comparisons was considered significant. Correlation and especially Bland Altman analyses (Bland \& Altman, 1986) of the resulting relative lobar perfusion between both approaches (hybrid approach vs. non-hybrid approach) were performed to test for good agreement of the results. An estimation for the required sample size was done, yielding a minimum sample size of 111 pairs when setting type I error to 0.05 and type II error to 0.2 with an expected mean of the difference of 0 , expected SD of differences of 2 and a maximum allowed difference between both methods of 5. Intraclass correlation coefficient was calculated. 


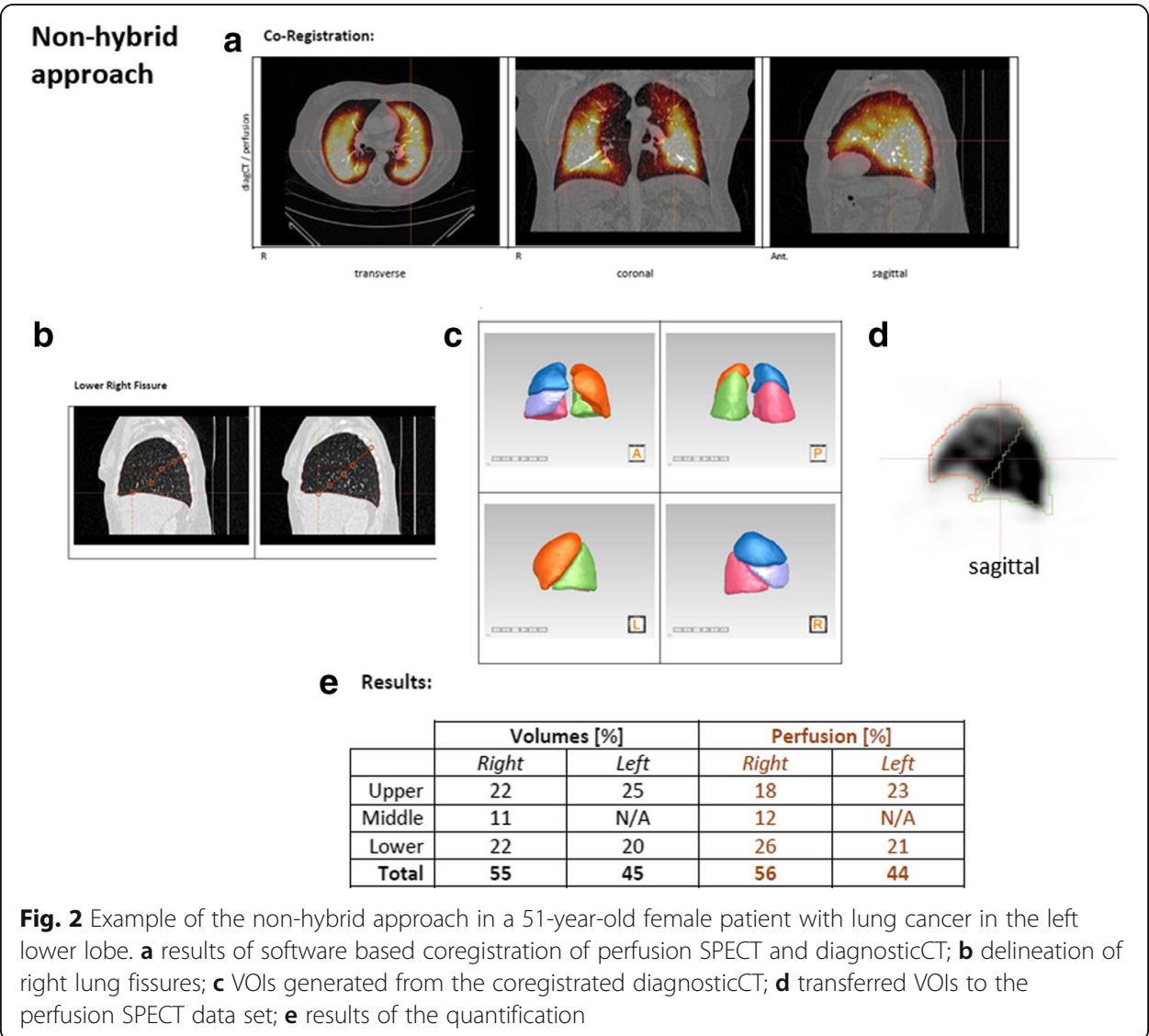

\section{Results}

\section{Lobar results}

Three hundred five lobes were quantified with both approaches. Processing time was 6:37 $\pm 0.55 \mathrm{~min}$ (hybrid) versus 6:34 $\pm 0: 51 \mathrm{~min}$ (non-hybrid) $(p=0.6)$ so no relevant difference between the two workflow procedures was found. Mean lobar value was $20.0 \pm 10.5 \%$ (range from 0 to 55\%) for hybrid and $20.0 \pm 10.6 \%$ (range from 0 to $58 \%$ ) for the non-hybrid approach, mean absolute difference was $1.31 \%$, no significant differences were found when analysing all lobe values pairwise using a t-test for paired samples $(p>0.9)$. Intraclass correlation coefficient was 0.9843 .

\section{Correlation and Bland Altman analysis}

Correlation was excellent for both approaches with $R=0.984$ and a slope of the regression line of $1.001(p<0.0001)$, see Fig. 3a. In the Bland Altman plot (see Fig. 3b) a mean value of the difference of $0 \%$ was found with lower and upper limits of $-3.67 \%$ and $3.67 \%$.

\section{Right lung}

Relative lobar perfusion of the right upper lobe was not significantly different $(p=0.15)$ between hybrid and non-hybrid approach $(18.4 \pm 10.0 \%$ (hybrid) vs. $18.0 \pm 10.0 \%$ (non-hybrid)). For the middle lobe relative lobar perfusion differed not significantly ( $p=$ 


\section{a}

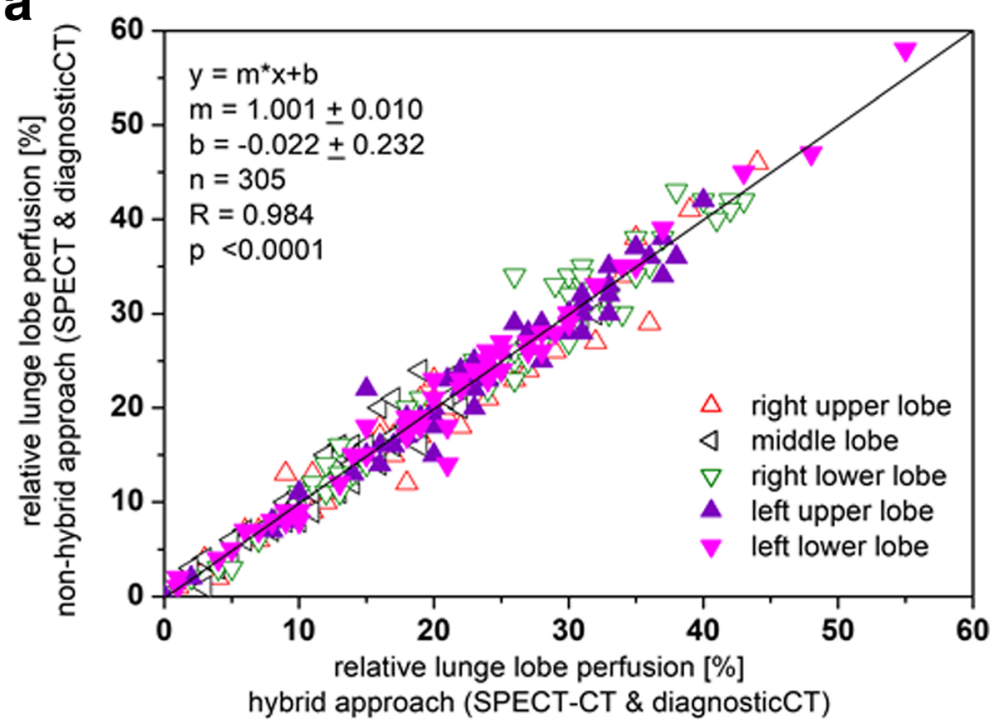

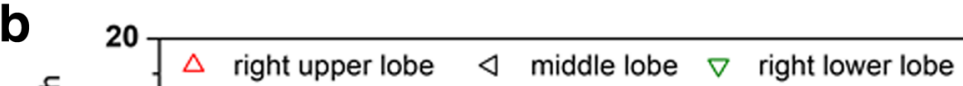

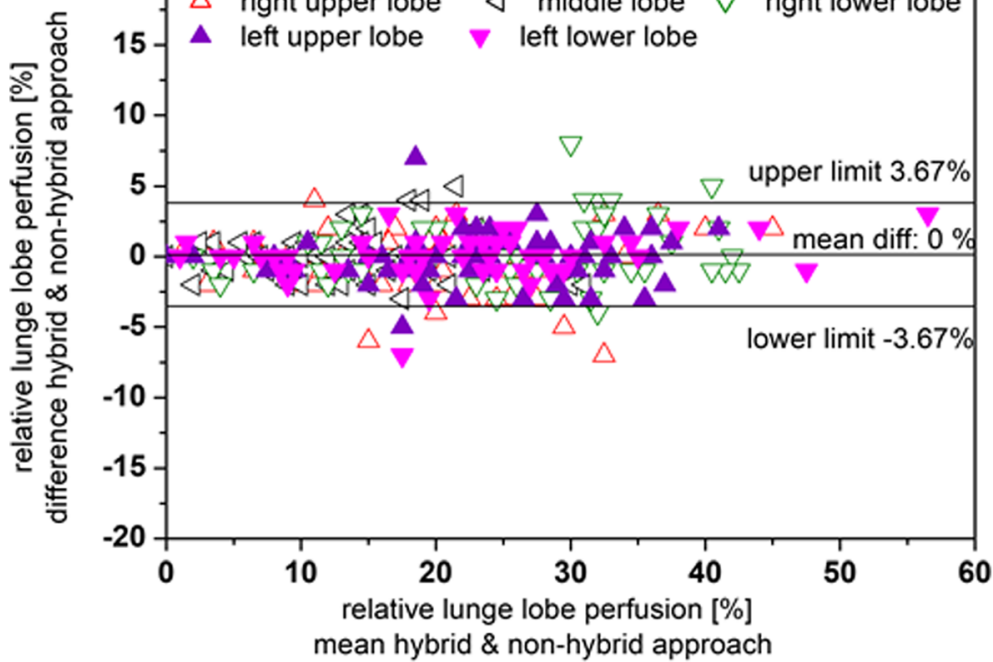

Fig. 3 a Correlation analysis of the relative lobar lung perfusion results between the non-hybrid and the hybrid approach. $\mathbf{b}$ Corresponding Bland Altman analysis

$0.50)$ between hybrid and non-hybrid approach (13.3 $\pm 6.6 \%$ (hybrid) vs. $13.5 \pm 6.6 \%$ (non-hybrid)). Relative lobar perfusion of the right lower lobe was not significantly different $(p=0.19)$ between hybrid and non-hybrid concept $(24.6 \pm 10.9 \%$ (hybrid) vs. $25.0 \pm 11.2 \%$ (non-hybrid)).

\section{Left lung}

Relative lobar perfusion of the left upper lobe was not significantly different $(p=0.57)$ between hybrid and non-hybrid method (23.0 $\pm 8.9 \%$ (hybrid) vs. $22.8 \pm 9.1 \%$ (non-hybrid)). For the left lower lobe relative lobar perfusion differed not significantly ( $p=$ 0.93 ) between hybrid and non-hybrid concept (20.7 $\pm 11.5 \%$ (hybrid) vs. $20.7 \pm 11.8 \%$ (non-hybrid)). 


\section{Secondary findings}

In only 2 of the 61 patients pulmonary embolism were found, those weren't described in the diagnostic CT's with contrast media 2-3 weeks before. We cannot distinguish between false negative CT's or whether the PE occurred in between. The only explanation we have for the low rate of PEs at all is that those with PE diagnosed in the primary CT did not get referred for lobar quantification.

\section{Discussion}

Accurate state-of-the-art preoperative assessment of pulmonary function is essential prior to surgical treatment in patients with pulmonary tumors (Knollmann et al., 2014, (Toney et al., 2014)) particularly in patients with borderline lung function (Brunelli et al., 2009). Loss of lung tissue may cause deterioration in pulmonary function and cardiopulmonary failure, leading to a loss of quality of life and, in worst case, death. The more precise the prediction of postoperative lung function is, the better patients can be selected to be or not to be eligible for surgery. 3D SPECT quantification of relative lobar lung function overcomes thereby the traditional quantification method using planar images. A CT based segmentation approach compared to a less precise planar method is effective and allowed in a previous analysis curative surgical treatment in 5 of 36 patients in which planar data would exclude surgery with 2 patients vice versa (Knollmann et al., 2014). The advantages of SPECT and SPECT/CT in V/Q scanning and its benefit for 3D quantification were also previously described by Roach et al. (Roach et al., 2010).

Undoubtedly hybrid SPECT/CT-machines are ideally suited to solve the problem of lobe delineation and perfusion quantification (Knollmann et al., 2014), but these are still limited in availability and often only suitable for low-dose CT (especially in first generation SPECT/CTs) requiring the use of a diagnostic CT to achieve precise lobar delineation. But virtually all patients with lung tumours have undergone a timely diagnostic CT before lung perfusion SPECT, in the present study availability rate was more than $97 \%$.

In this study the patient's diagnostic CT was used for lobar delineation and the low-dose CT was used as an intermediate bridge for coregistration of the diagnostic $\mathrm{CT}$ to the perfusion SPECT. Therefore the questions arose, whether the software could be used without the low-dose CT, what outcome this would have on results and whether the processing time would increase.

Unexpectedly using only the lung perfusion SPECT and diagnostic CT results were statistically not less accurate than using the additional low-dose-CT data from the SPECT/CT-machine as bridge for the coregistration. We found an excellent correlation between the results from the hybrid and the non-hybrid approach (Fig. 3a) and extremely narrow Bland Altman limits (Fig. 3b) proving the good agreement of both approaches. Additionally, unexpectedly the processing time was the same for both approaches, since data handling and processing and the fissure definition are the time consuming elements of the procedures, and those are in both approaches virtually the same.

Therefore the non-hybrid approach is from our point of view not inferior to the hybrid approach and widespread 3D quantification of lobar lung function can be realised without the need for costly investment of SPECT/CT systems for this clinical question. 
Nevertheless, since in our approach the lung SPECT scans are also used to search for pulmonary embolism - PE was diagnosed in 2 of the 61 patients in the cohort - we will not resign the low dose CTs since $0.66 \mathrm{mSv}$ are from our point of view negligible and the low-dose CTs help us interpreting the SPECT scans by increasing specificity (Gutte et al., 2009).

\section{Conclusions}

No relevant differences for 3D quantification of relative lung perfusion in preoperative patients with pulmonary tumours were found when comparing a hybrid and a non-hybrid scanner approach. Using sophisticated software and the almost always available diagnostic full-dose CT accurate and reliable results can be obtained from conventional SPECT data without increasing the processing time.

\section{Abbreviations}

CT: X-ray Computed Tomography; CTDI: Computed Tomography Dose Index; DLP: Dose-Length Product; MAA: Macro Aggregated Albumin; MBq: MegaBecquerel; mGy: milliGray; mSv: milliSievert; SD: Standard Deviation; SPECT: Single Photon Emission Computed Tomography

\section{Acknowledgments}

We thank the team of S. Peterson-Zysk for their great support and realisation of SPECT/CT investigations.

\section{Availability of data and materials}

Not shared because of legal responsibility to respect participants' rights to privacy and to protect their identity.

\section{Authors' contributions}

DK contributed to conception and design as well as analysis and acquisition of all patient data and drafted the manuscript. JA contributed to analysis of data and revised the manuscript. WMS analysed and interpreted the data and revised and approved the manuscript for submission. All authors read and approved the final manuscript.

\section{Ethics approval and consent to participate}

All procedures performed in studies involving human participants were in accordance with the ethical standards of the institutional and/or national research committee and with the 1964 Helsinki declaration and its later amendments or comparable ethical standards. The study has been approved by the ethical committee of the Medical Faculty of the Ruhr-University-Bochum (AZ2017245). All subjects signed an informed consent form for the investigation and data analysis using cloud software.

\section{Competing interests}

Daniela Knollmann: Lecturer (Curium).

Jerome Avondo: Employee HERMES Medical Solutions.

Wolfgang M. Schaefer: Travel expenses (Cyclomedica, Siemens). Lecturer (Siemens, Curium). Advisory Board (Covidien, Curium).

\section{Publisher's Note}

Springer Nature remains neutral with regard to jurisdictional claims in published maps and institutional affiliations.

\section{Author details}

${ }^{1}$ Kliniken Maria Hilf, Nuclear Medicine, Viersener Str. 450, 41063 Moenchengladbach, Germany. ${ }^{2}$ HERMES Medical

Solutions, Skeppsbron 44, 11130 Stockholm, Sweden.

Received: 23 March 2018 Accepted: 12 June 2018

Published online: 10 September 2018

\section{References}

Bland JM, Altman DG (1986) Statistical methods for assessing agreement between two methods of clinical measurement. Lancet 1:307-310

Bolliger CT, Perruchoud AP (1998) Functional evaluation of the lung resection candidate. Eur Respir J 11:198-212

Bria WF, Kanarek DJ, Kazemi H (1983) Prediction of postoperative pulmonary function following thoracic operations. Value of ventilation-perfusion scanning. JThorac. Cardiovasc Surg 86:186-192

Brunelli A, Charloux A, Bolliger CT, Rocco G, Sculier J-P, Varela G et al (2009) ERS/ESTS clinical guidelines on fitness for radical therapy in lung cancer patients (surgery and chemo-radiotherapy). Eur Respir J 34:17-41

Giordano A, Calcagni ML, Meduri G, Valente S, Galli G (1997) Perfusion lung scintigraphy for the prediction of postlobectomy residual pulmonary function. Chest 111:1542-1547 
Gutte H, Mortensen J, Jensen CV, Johnbeck CB, von der Recke P, Petersen CL et al (2009) Detection of pulmonary embolism with combined ventilation-perfusion SPECT and low-dose CT: head-to-head comparison with multidetector CT angiography. J Nucl Med 50:1987-1992

Imaeda T, Kanematsu M, Asada S, Seki M, Matsui E, Doi H et al (1995) prediction of pulmonary function after resection of primary lung cancer. Utility of inhalation-perfusion SPECT imaging. Clin Nucl Med 20:792-799

Knollmann D, Meyer A, Noack F, Schaefer WM (2015) Preoperative assessment of relative pulmonary lobar perfusion fraction in lung cancer patients. A rather simple three-dimensional CT-based vs. planar image-derived quantification. Nuklearmedizin 54:178-182

Markos J, Mullan BP, Hillman DR, Antico VF et al (1989) Preoperative assessment as a predictor of mortality and morbidity after lung resection. Am Rev Respir Dis 139:902-910

Mende T, Orlick M, Fischbeck O, Neef H (1990) Risk assessment of bronchial cancer surgery using quantitative lung perfusion scintigraphy. Nuklearmedizin 29:274-277

Ohno Y, Koyama H, Nogami M, Takenaka D, Onishi Y, Matsumoto K et al (2011) State-of-the-art radiological techniques improve the assessment of postoperative lung function in patients with non-small cell lung cancer. Eur J Radiol 77: 97-104

Roach PJ, Schembri GP, Ho Shon IA, Bailey EA, Bailey DL (2010) SPECT/CT in V/Q scanning. Semin Nucl Med 40:455-466

Suga K, Kawakami Y, Zaki M, Yamashita T, Shimizu K, Matsunaga N (2004) Clinical utility of co-registered respiratorygated (99m)Tc-Technegas/MAA SPECT-CT images in the assessment of regional lung functional impairment in patients with lung cancer. Eur J Nucl Med Mol Imaging 31:1280-1290

Toney LK, Wanner M, Miyaoka RS, Alessio AM, Wood DE, Vesselle H (2014) Improved prediction of lobar perfusion contribution using technetium-99m-labeled macroaggregate of albumin single photon emission computed tomography/computed tomography with attenuation correction. J Thorac Cardiovasc Surg 148:2345-2352

Win T, Tasker AD, Groves AM, White C, Ritchie AJ, Wells FC et al (2006) Ventilation-perfusion scintigraphy to predict postoperative pulmonary function in lung cancer patients undergoing pneumonectomy. Am J Roentgenol 187: $1260-1265$

Wu MT, Pan HB, Chiang AA, Hsu HK, Chang HC, Peng NJ et al (2002) Prediction of postoperative lung function in patients with lung cancer: comparison of quantitative $C T$ with perfusion scintigraphy. Am J Roentgenol 178:667-672

Submit your manuscript to a SpringerOpen ${ }^{\odot}$ journal and benefit from:

- Convenient online submission

- Rigorous peer review

Open access: articles freely available online

- High visibility within the field

Retaining the copyright to your article 